\title{
CONF-95/155--46
}

Note: This is a preprint of a paper being submitted for publication. Contents of this paper should not be quoted nor referred to without permission of the author(s).

1995 MRS FALL MEETING

\section{Optical Properties of Multicomponent Antimony-Silver Nanoclusters Formed in Silica by Sequential Ion Implantation}

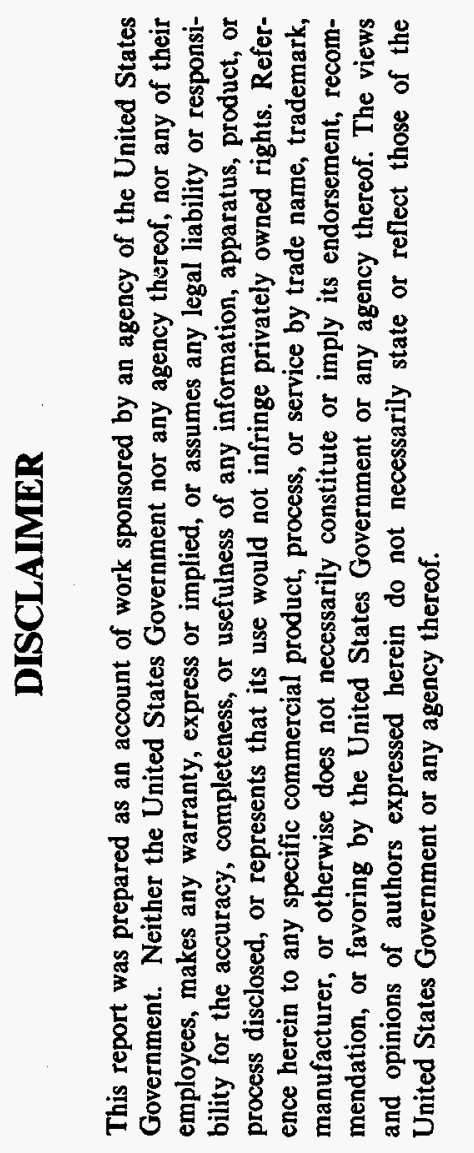

\author{
R. A. Zuhr \\ Oak Ridge National Laboratory \\ Oak Ridge, TN \\ R. H. Magruder, III, and T. S. Anderson \\ Vanderbilt University \\ Nashville, TN \\ "The submitted manuscript has been \\ anthored by $a$ contractor of the U.S. \\ Government under contract No. DE- \\ AC05-840R21400. Accordingly, the \\ U.S. Government retains a nonexclusive, \\ royalty-free license to publish or \\ royalty-free
reproduce the published form of this \\ contribution, or allow others to do so, \\ for U.S. Government purposes." \\ Prepared by the \\ Oak Ridge National Laboratory \\ Oak Ridge, Tennessee 37831 \\ managed by \\ MARTIN MARIETTA ENERGY SYSTEMS, INC. \\ for the \\ U.S. DEPARTMENT OF ENERGY \\ under contract DE-AC05-84OR21400
}

November 1995 


\title{
OPTICAL PROPERTIES OF MULTI-COMPONENT ANTIMONY-SILVER NANOCLUSTERS FORMED IN SILICA BY SEQUENTIAL ION IMPLANTATION
}

\author{
R.A. ZUHR*, R.H. MAGRUDER, III**, AND T.S. ANDERSON** \\ *Solid State Division, Oak Ridge National Laboratory, Oak Ridge, TN 37831 \\ **Vanderbilt University, Nashville, TN 37235
}

\begin{abstract}
The linear and nonlinear optical properties of nanometer dimension metal colloids embedded in a dielectric depend explicitly on the electronic structure of the metal nanoclusters. The ability to control the electronic structure of the nanoclusters may make it possible to tailor the optical properties for enhanced performance. By sequential implantation of different metal ion species multi-component nanoclusters can be formed with significantly different optical properties than single element metal nanoclusters. We report the formation of multi-component $\mathrm{Sb} / \mathrm{Ag}$ nanoclusters in silica by sequential implantation of $\mathrm{Sb}$ and Ag. Samples were implanted with relative ratios of $\mathrm{Sb}$ to Ag of 1:1 and 3:1. A second set of samples was made by single element implantations of $\mathrm{Ag}$ and $\mathrm{Sb}$ at the same energies and doses used to make the sequentially implanted samples. All samples were characterized using RBS and both linear and nonlinear optical measurements. The presence of both ions significantly modifies the optical properties of the composites compared to the single element nanocluster glass composites. In the sequentially implanted samples the optical density is lower, and the strong surface plasmon resonance absorption observed in the Ag implanted samples is not present. At the same time the nonlinear response of the these samples is larger than for the samples implanted with $\mathrm{Sb}$ alone, suggesting that the addition of Ag can increase the nonlinear response of the $\mathrm{Sb}$ particles formed. The results are consistent with the formation of multi-component Sb/Ag colloids.
\end{abstract}

\section{INTRODUCTION}

Sequential ion implantation of different elements has been shown to alter the composition of the metal colloids that are formed, as well as to modify the guest host interaction, in metal-glass composite optical materials. [1-4] Composition engendered changes in the electronic structure of the metal colloids can result in changes to both the linear and nonlinear optical properties of the composite that are not possible with colloids composed of single elements.[1-4]

The linear and nonlinear optical properties of these metal colloid-glass composites can be described using effective medium theory for small non interacting particles in a dielectric.[5] An increase in the nonlinear response of the composite near the surface plasmon resonance (SPR) frequency is expected with increasing volume fraction of metal. This enhancement is due to local field effects and is a consequence of dielectric confinement.

The linear response for colloids with diameters less than $\lambda / 20$, where $\lambda$ is the wavelength of the incident radiation, is reasonably described by effective medium theory in the electric dipole approximation[6] and is given by

$$
\alpha=\frac{18 \pi \mathrm{n}_{\mathrm{d}}^{3}}{\lambda} \cdot \frac{\mathrm{p} \varepsilon_{2}}{\left[\varepsilon_{1}+2 \mathrm{n}_{\mathrm{d}}^{2}\right]^{2}+\varepsilon_{2}^{2}},
$$

where $\alpha$ is the absorption coefficient, $\varepsilon(\lambda)=\varepsilon_{1}+i \varepsilon_{2}$ is the dielectric constant of the metal, $p$ is the volume fraction of the metal particles, and $n_{d}$ is the index of refraction of the dielectric host. The absorption is expected to exhibit a peak at the surface plasmon resonance frequency for which the condition $\varepsilon_{1}+2 n_{d}^{2}=0$ is met. The surface plasmon resonance frequency depends on the electronic properties of the metal colloids and on the index of refraction of the host dielectric, $\mathrm{n}_{\mathrm{d}}$. The third order nonlinear susceptibility, $\chi_{\text {eff }}^{(3)}$, can be expressed as 


$$
\chi_{\text {eff }}^{(3)}=p f_{c}^{2}(\omega)\left|f_{2}(\omega)\right|^{2} \chi_{m}^{(3)} \text { with } f_{c}(\omega)=\frac{3 n_{d}^{2}}{\varepsilon_{1}+2 n_{d}^{2}}
$$

and where $f_{c}(\omega)$ is the local field factor and $\chi_{m}^{(3)}$ is the nonlinear susceptibility of the metal colloids. The index of refraction and the intensity dependent term are related to the above quantities by[7]

$$
\mathrm{n}=\mathrm{n}_{\mathrm{o}}+\mathrm{n}_{2} \mathrm{I} \text { and } \mathrm{n}_{2}=\frac{12 \pi}{\mathrm{n}_{\mathrm{o}}} \operatorname{Re}\left[\chi_{\text {eff }}^{(3)}\right] \text {, }
$$

where $n_{0}$ is the linear index of refraction and $n_{2}$ is the intensity dependent component. From equation (2), a potentially large enhancement of the effective nonlinear susceptibility and in turn $\mathrm{n}_{2}$ is possible near or at the surface plasmon resonance frequency due to local field effects.

Here we report on the optical response of nanometer dimension metal colloids formed by sequential implantation of $\mathrm{Ag}$ and $\mathrm{Sb}$. $\mathrm{Ag}$ and $\mathrm{Sb}$ were chosen because of their fundamentally different dielectric properties and their observed nonlinear response in metal nanocluster glass composites. $[8,9,10]$

\section{EXPERIMENT}

First $\mathrm{Ag}$ and then $\mathrm{Sb}$ ions were sequentially implanted in Corning 7940 high purity silica substrates at energies that overlaid the depths of implantation for each metal. The $\mathrm{Ag}$ ions were implanted at $305 \mathrm{keV}$ while the $\mathrm{Sb}$ ions were implanted at $320 \mathrm{keV}$, with substrate temperatures of $\sim 270^{\circ} \mathrm{K}$ and current densities of $\sim 2 \mu \mathrm{amps} / \mathrm{cm}^{2}$ for both ions. Doses were 3 and $6 \times 10^{16}$ ions $/ \mathrm{cm}^{2}$ for the $\mathrm{Ag}$ and 6 and $9 \times 10^{16}$ ions $/ \mathrm{cm}^{2}$ for the Sb implantations. The sequentially implanted samples have a total nominal dose of $12 \times 10^{16} \mathrm{ions} / \mathrm{cm}^{2}$ with relative ratios of $\mathrm{Ag}$ to $\mathrm{Sb}$ of $3 / 9$ and $6 / 6$. Single element implanted samples were also fabricated for comparison. These samples were implanted to nominal doses of 3 and $6 \times 10^{16}$ ions $/ \mathrm{cm}^{2}$ for Ag and 6 and $9 \times 10^{16}$ ions $/ \mathrm{cm}^{2}$ for $\mathrm{Sb}$ using the same experimental conditions as for the sequentially implanted samples.

Rutherford backscattering (RBS) with $2.3 \mathrm{MeV} \mathrm{He}++$ ions at 160 degrees was used to measure the depth profiles of the implanted species. Samples for transmission electron microscopy (TEM) were prepared in a planar configuration using conventional techniques reported in prior work.[1] Standard bright field (BF) imaging was used to observe the implanted layer and selected area electron diffraction was used to analyze the structure of the metal colloids formed in the silica wafers.

Linear optical absorption measurements were made at room temperature in air from 900 to $200 \mathrm{~nm}$ using a dual beam spectrometer (Cary 5). All samples were measured using an unimplanted sample in the reference beam. Absorption spectra were measured at three different positions on each sample and the scatter in the absorption coefficient at these different positions was less than $2 \%$. The nonlinear index of refraction was measured at $596 \mathrm{~nm}$ using the Z-scan method described previously. The laser used for these experiments was a cavity dumped tunable dye laser with a $\sim 6$ ps pulse duration operated at $3.8 \mathrm{MHz}$. The average power was $250 \mathrm{~mW}$ in the $\mathrm{TEM}_{00}$ mode and the peak irradiance for a focal spot $25 \mathrm{~mm}$ in radius was $-4.5 \times 10^{8} \mathrm{~W} / \mathrm{cm}^{2}$.

\section{RESULTS}

From RBS measurements of the sequentially implanted samples the metal depth distribution as a function of distance from the surface was found to be skewed gaussian in shape with a shoulder at $\sim 0.07$ microns and a peak in the distribution at $\sim 0.14$ microns. The RBS spectra for the single element implantations are approximately gaussian in shape with the peaks at $\sim 0.13$ microns for the $\mathrm{Ag}$ and $\sim 0.15$ microns for the $\mathrm{Sb}$ samples. The full width at half maximum (FWHM) is $\sim 0.2$ microns for the Sb implanted sample and $\sim 0.09$ microns for the $\mathrm{Ag}$ implanted sample. 


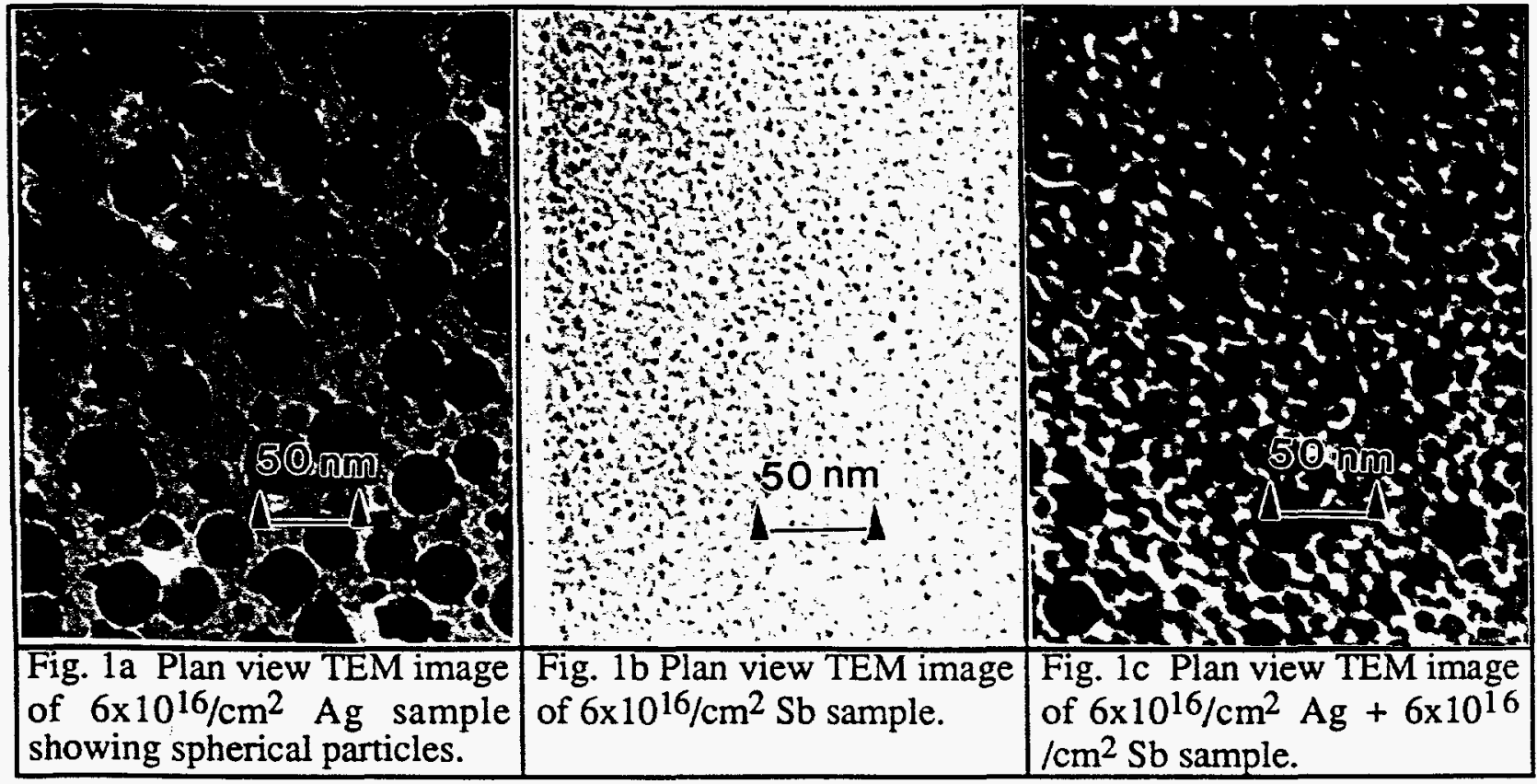

Bright field images for the $6 \mathrm{Ag}$ and $6 \mathrm{Sb}$ single element samples are shown in Figure 1a,b. The 6Ag sample has spherical particles, while the $6 \mathrm{Sb}$ sample forms smaller colloids that are irregularly shaped and only approximately spherical. A plan view TEM image for the $6 \mathrm{Ag} / 6 \mathrm{Sb}$ sample is shown in Figure 1c. In this case the implanted ions again form colloids that are approximately spherical with irregularly shaped surfaces. An increase in colloid size is observed for an increase in the amount of implanted Ag.

Selected area diffraction patterns from the Ag samples reveal distinct metallic crystal structures in the implanted layer. The features in the polycrystalline ring patterns are representative of the face centered cubic (FCC) Ag lattice. For the Sb implanted samples selected area diffraction patterns indicate that the colloids formed are amorphous in nature. Calculated d-spacings for the $6 \mathrm{Ag} / 6 \mathrm{Sb}$ sample are inconsistent with those expected from single element colloids of either Ag or Sb. Calculated d-spacings for the $6 \mathrm{Ag} / 6 \mathrm{Sb}$ sample are 2.58 , $2.27,1.43$, and $1.32 \AA$. The colloids formed in the $3 \mathrm{Ag} / 9 \mathrm{Sb}$ sample are amorphous.

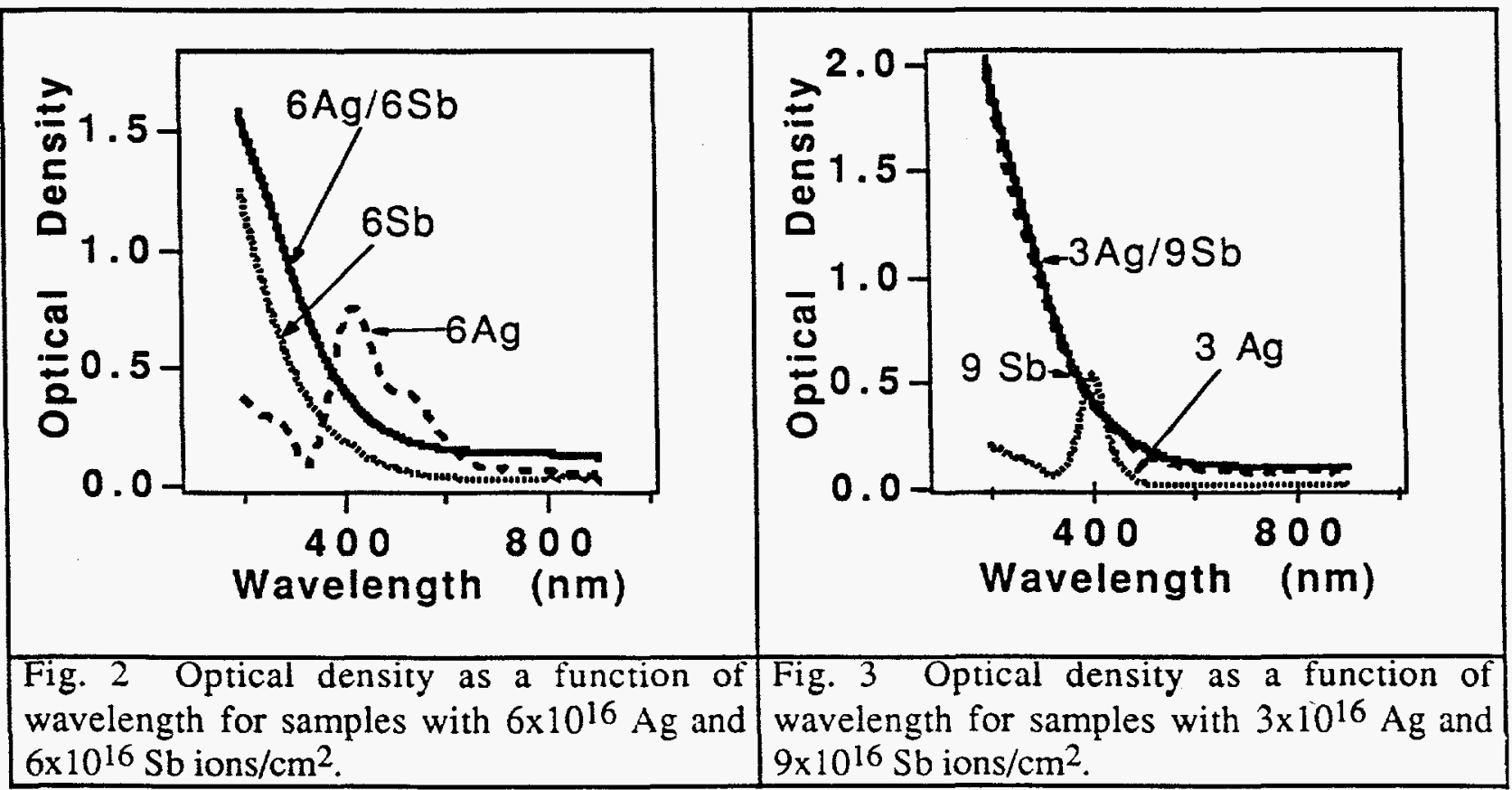


Figures 2 and 3 show the optical absorption spectra as a function of photon wavelength for each of the sequentially implanted samples and their respective single element counterparts. The absorption in both the $3 \mathrm{Ag} / 9 \mathrm{Sb}$ and the $6 \mathrm{Ag} / 6 \mathrm{Sb}$ samples increases with decreasing wavelength and shows no distinguishing features.

For the single element implantations the absorption spectra for the Ag implanted samples are dominated by an absorption peak at $\sim 400 \mathrm{~nm}$ that increases in magnitude and red shifts with increasing nominal dose. The $6 \times 10^{16} \mathrm{Ag}$ sample has an absorption peak centered at $\sim 420 \mathrm{~nm}$ and a shoulder at $\sim 520 \mathrm{~nm}$. For the Sb implanted samples the absorption increases with decreasing wavelength. The absorption in these samples has qualitatively the same dependence on photon wavelength as the sequential samples, and increases in total absorption with increasing dose.

Readily observable is that none of the sequentially implanted spectra match the spectra observed for Ag single element implanted samples. The peak at $\sim 400 \mathrm{~nm}$ and shoulder at 520 $\mathrm{nm}$ are not observed in any of the sequentially implanted samples.

Table I lists the linear and nonlinear optical coefficients for all samples measured at $596 \mathrm{~nm}$. These coefficients were calculated using a thickness of 0.15 microns. The nonlinear coefficients were calculated using the formalism of reference [11].

For the sequentially implanted samples, the absorption and $\mathrm{n}_{2}$ at $596 \mathrm{~nm}$ increase with increasing Ag dose. Similar behavior is observed for the Ag implanted samples. For the $6 \mathrm{Ag}$ sample a saturated nonlinear absorption is observed, as indicated by the negative sign for the $\beta$ value in the table. The values of $\mathrm{n}_{2}$ for the $\mathrm{Sb}$ implanted glasses also increase with increasing dose. However neither the $\mathrm{Sb}$ nor the $\mathrm{Ag} / \mathrm{Sb}$ samples have a measurable nonlinear absorption at this wavelength.

Table I

Linear and Nonlinear Optical Coefficients @ 596 nm

\begin{tabular}{|c|c|c|c|}
\hline Sample & $\begin{array}{c}\text { Absorption } \\
\text { Coefficient }\left(\mathrm{cm}^{-1}\right)\end{array}$ & $\begin{array}{c}\mathrm{n} 2 \\
(\mathrm{~cm} 2 / \mathrm{W})\end{array}$ & $\begin{array}{c}\beta \\
(\mathrm{cm} / \mathrm{W})\end{array}$ \\
\hline $3 \mathrm{Ag} / 9 \mathrm{Sb}$ & $1.82 \times 10^{4}$ & $1.3 \times 10^{-9}$ & N.D. \\
\hline $6 \mathrm{Ag} / 6 \mathrm{Sb}$ & $2.45 \times 10^{4}$ & $1.5 \times 10^{-9}$ & N.D. \\
\hline $3 \mathrm{Ag}$ & $0.14 \times 10^{4}$ & N.D. & N.D. \\
\hline $6 \mathrm{Ag}$ & $3.38 \times 10^{4}$ & $0.9 \times 10^{-9}$ & $-0.2 \times 10^{-4}$ \\
\hline $6 \mathrm{Sb}$ & $0.56 \times 10^{4}$ & $0.4 \times 10^{-9}$ & N.D. \\
\hline $9 \mathrm{Sb}$ & $1.40 \times 10^{4}$ & $0.9 \times 10^{-9}$ & N.D. \\
\hline
\end{tabular}

\section{DISCUSSION}

For the single element implants the $\mathrm{Sb}$ has a broader ion distribution than the Ag. The projected ranges of the $\mathrm{Ag}$ ions, 0.146 microns, and $\mathrm{Sb}$ ions, 0.143 microns, from TRIM calculations agree reasonably well with the results observed in the implanted samples. While each of the sequentially implanted sample ion distribution profiles is slightly different from the other, as well as from the single element ion distributions, the majority of the two different ions species overlap in the sequential samples.

Crystalline colloids are formed in the $3 \mathrm{Ag}$ and $6 \mathrm{Ag}$ samples, while amorphous particles are formed in the Sb implants. In the sequentially implanted samples the structure of the colloids depends on the ratio of $\mathrm{Ag}$ to $\mathrm{Sb}$. The theoretical d-spacings for the mineral dyscrasite ( $\mathrm{Ag}_{3} \mathrm{Sb}$ ) are $2.60,2.29,1.36$, and $1.30 \AA$. This agrees, within error, with the calculated d-spacings of the 
6Ag/6Sb sample. The RBS measurements for the single element implanted samples show that the FWHM is approximately twice as large for the implanted Sb as it is for the Ag. The resulting difference in concentration could account for the formation of dyscrasite in the implanted layer. For the $3 \mathrm{Ag} / 9 \mathrm{Sb}$ sample the colloids are amorphous, suggesting that the $\mathrm{Sb}$ dramatically changes the nature of the previously implanted Ag, which forms FCC metallic colloids in the single element implantations.

While part of the absorption in the ultraviolet may be attributed to defect centers $[12,13]$, based on prior work we expect that the absorption due to this mechanism will be dominated by the presence of the metal colloids.[1-4]

The optical spectra of the glasses implanted only with $\mathrm{Ag}$ exhibit characteristic absorption due to the surface plasmon resonance of nanometer dimension Ag colloids. [4, 14] We attribute the peak at $400 \mathrm{~nm}$ in the Ag implanted samples to Ag colloids $<20 \mathrm{~nm}$ in diameter, and the red shift of this peak with increasing Ag dose to an increase in size of these Ag colloids. $[4,15]$ We attribute the shoulder at $520 \mathrm{~nm}$ in the $6 \times 10^{16} \mathrm{Ag}$ ions $/ \mathrm{cm}^{2}$ sample to an additional higher order absorption term in Mie theory, i.e., the quadrupole term.[15]

For the single element $\mathrm{Sb}$ implanted samples there are no indications of any surface plasmon resonance absorption in the optical absorption spectra. We attribute the increasing absorption with decreasing photon wavelength in these samples to the formation of $\mathrm{Sb}$ nanometer dimension colloids. The wavelength dependence is qualitatively the same for all the Sb samples, and the magnitude of the absorption increases with increasing dose. The increase in absorption with increasing dose is due to an increase in metal volume fraction. Both of these observations are in accord with equation (1) and prior results. [8] The dielectric properties of the Sb nanoclusters are such that the condition $\varepsilon_{1}+2 n_{d}^{2}=0$ is not satisfied $[9,10]$ and therefore these samples do not exhibit a distinct surface plasmon resonance absorption feature. However the spectra do exhibit the approximate $1 / \lambda$ dependence and the increase in optical density with increasing metal volume fraction that are expected from equation (1).

The optical properties of the sequentially implanted samples are clearly altered from their single element counterparts. The presence of the absorption due to the surface plasmon resonance of the $\mathrm{Ag}$ colloids is no longer observed. From equation (1) the volume fraction of the metal present and the dielectric function, $\varepsilon(\lambda)=\varepsilon_{1}+\varepsilon_{2}$, of the metal clusters are expected to impact the absorption explicitly. Equation (1) predicts an increase in the absorption based on the increase in the volume fraction of metal ions. This is not observed in the $6 \mathrm{Ag} / 6 \mathrm{Sb}$ sample. We have increased the volume fraction of the metal ions by a factor of 2 over the single element implanted $6 \mathrm{Ag}$ sample, yet the linear absorption decreases significantly. For non-interacting colloids we would expect, from effective medium theory, that the absorption would be a simple superposition of the absorption from the $6 \mathrm{Ag}$ and $6 \mathrm{Sb}$ samples. This assumption yields absorption that is a factor of 1.6 larger than what is actually observed for the $6 \mathrm{Ag} / 6 \mathrm{Sb}$ sample. The sequentially implanted samples have a wavelength dependence similar to the Sb implanted samples, but as noted above, behave differently than the superposition of the individual components. These differences indicate that the presence of these two ions produces unique multi-component colloids whose characteristics depend on the $\mathrm{Ag} / \mathrm{Sb}$ ratio.

We can conclude from the TEM and optical measurements that the multi-component colloids formed have a significantly different electronic structure than the elemental colloids. This difference results in the changes observed in the linear optical properties.

The nonlinear index of refraction, $\mathrm{n}_{2}$, at $596 \mathrm{~nm}$ increases with the sequential implantation of $\mathrm{Ag}$ with $\mathrm{Sb}$. The value of $\mathrm{n}_{2}$ for the $6 \mathrm{Ag} / 6 \mathrm{Sb}$ sample is $\sim 4$ times greater than $\mathrm{n}_{2}$ for the $6 \mathrm{Sb}$ sample and $-70 \%$ greater than that for the $6 \mathrm{Ag}$ sample. Nonlinear absorption is no longer detected, and the linear absorption decreases $-25 \%$ compared to the $6 \mathrm{Ag}$ sample. Significantly, this increase in $\mathrm{n}_{2}$ occurs without the presence of a detectable surface plasmon resonance and with a decrease in total absorption. As observed in the preceding paragraphs, the volume fraction of metal ions has increased, but this cannot account for the changes in the nonlinear response observed. In addition, nonlinear absorption is no longer detectable in the $6 \mathrm{Ag} / 6 \mathrm{Sb}$ sample. This is the opposite of what would be expected if the volume fraction of metal ions was the dominant factor. In the case of the $3 \mathrm{Ag} / 9 \mathrm{Sb}$ sample the value for $\mathrm{n}_{2}$ is $\sim 50 \%$ larger than that for the $3 \mathrm{Ag}$ and the $9 \mathrm{Sb}$ samples. These results indicate that the electronic structure of the 
multi-component colloids formed has resulted in significant changes in $\varepsilon(\lambda)$ and $\chi_{\mathrm{m}}^{(3)}$ of the colloids.

A significant problem with metal colloid-glass composites for device applications is that both the linear and nonlinear absorption in general increase near the surface plasmon resonance frequency. This increase in absorption has deleterious effects on the figures of merit for devices operating near the SPR frequency for these materials.[16, 17] Even away from the SPR frequency, linear and nonlinear absorption can limit device potential. In this paper we have shown that the properties of the metal nanoclusters formed can be significantly altered by sequential implantation of multiple elements. In particular, $\mathrm{n}_{2}$ can be increased while both the linear and nonlinear absorption are decreased in comparison to single element colloids. This ability to form unique metal colloid-glass composites that have larger nonlinear responses, while at the same time exhibiting smaller absorption losses, could significantly improve the feasibility of these materials for optical devices.

\section{ACKNOWLEDGMENTS}

The authors acknowledge the support of the Army Research Office under grant DAAH04-93G-0123 and DAAH04-94-G-0147 and the Division of Materials Science, U.S. Department of Energy under contract DE-AC05-84OR21400 with Lockheed Martin Energy Systems, Inc. We thank Prof. Richard Haglund for the use of his laboratory for the nonlinear measurements.

\section{REFERENCES}

1. R. H. Magruder,III, J.E. Wittig and R.A. Zuhr, J. Non Cryst. Solids, 163 (1993) 162.

2.R.A. Zuhr, R.H. Magruder,III and J.E. Wittig, Mat. Res. Soc. Sym. Proc., 316 (1994) 457 .

3. R.H. Magruder,III, R.A. Zuhr and D. O. Osborne Nucl. Instr. and Meth. B. 99 (1995) 590.

4. T.S. Anderson, R.H. Magruder,III, R.A. Zuhr and J.E. Wittig, in press J. of Electronic Materials.

5. C. Flytzanis, F. Hache, M.C. Klein, D. Ricard and Ph. Roussignol, Progress in Optics, 29 (1991) 321 and references there in.

6. C.F. Bohren and D.R. Huffman, Absorption and Scattering of Light by Small Particles, John Wiley and Sons, New York (1983).

7. M.J. Weber, D. Milam and W.L. Smith, Optical Eng., 17 (1978) 463.

8. R.H. Magruder,III, T.S. Anderson, R.A. Zuhr and D.K. Thomas, accepted Nuc. Inst. Methods B.

9. J. Alan Creighton and D.G. Eadon, Chem. Soc. Faraday Trans., 87 (1991) 3881.

10. Z. Pan, S.H. Morgan, D.O. Henderson, S. Park, R.A. Weeks, R.H. Magruder,III and R. A. Zuhr, in press, J. Opt. Materials.

11. M. Sheik-Bahae, A. A. Said and E. W. VanStryland, Opt. Lett. 14 (1989) 955.

12. G. W. Arnold and P. Mazzoldi , Ion Beam Modification of Insulators, P. Mazzoldi and G.W.Amold, eds. Elsevier, Amsterdam(1987)

13. A recent review is given by R. A. Weeks, Materials Science and Technology, vol. 9, J. Zarzychi, ed., VCH, Weinheim (1991).

14. R.A. Wood, P.D. Townsend, N.D. Skelland, D.E. Hole, J. Barton and C.N. Afonso, J. Appl. Phys., 74 (1993) 5754.

15. M. Vollmer and U. Kreibig, Nuclear Physics Concepts in the Study of Atomic Cluster Physics, R. Schmidt, H.O. Lutz and R. Dreizler, eds., Springer - Verlag, Berlin , 1992.

16. V. Mizrahi, K.W. DeLong, G.I. Stegeman, M.A. Salfi and M.J. Andrejcu, Opt. Lett., 14 (1989) 1140.

17. G.I. Stegeman and R.H. Stolen, J. Opt. Soc. Am. B6 (1989) 652. 